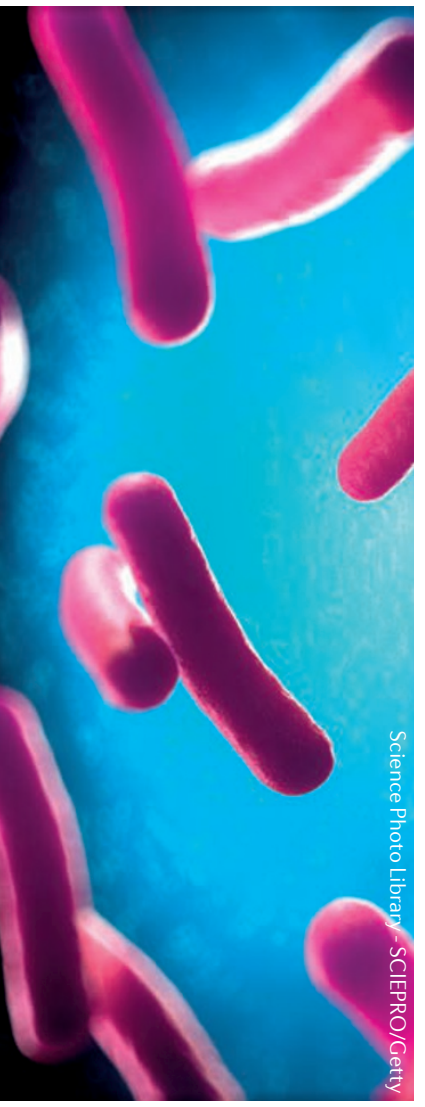

\title{
STONES
}

\section{Gut microbiome is unique in kidney stone disease}

Individuals with kidney stones have a unique gut microbiome (GMB) compared with those without kidney stones, according to a small pilot study.

Composition of the GMB has been associated with obesity, type 2 diabetes and cardiovascular disease, but little is known about the relationship between the GMB and kidney stone disease. "The role of gut microbiota in diseases linked to the metabolic syndrome provides motivation to investigate the relationship between the microbiota and kidney stone disease," say the authors of the new paper.

Stern and co-workers' proof-of-principle pilot study included 29 individuals, 23 with kidney stones and six without. GMB analysis - using next-generation sequencing to analyse 16 s ribosomal RNA marker gene sequences - was performed on faecal samples collected after a $6 \mathrm{~h}$ fast. The researchers identified 178 bacterial genera in the samples using this method. They found that the five most abundant enterotypes within each group made up $>50 \%$ of the bacterial abundance found. Bacteroides spp. were 3.4 times more abundant in patients with kidney stones than in controls $(34.9 \%$ versus $10.2 \% ; P=0.001)$, whereas Prevotella spp. were 2.8 times more abundant in the control group than in those with kidney stones (34.7\% versus $12.3 \% ; P=0.005)$. No significant differences were found in bacterial abundance between samples from patients with uric acid stones and those with calcium-based stones.

The researchers also investigated correlations between $24 \mathrm{~h}$ urine parameters and bacterial abundance in 11 patients with kidney stones. Among the bacterial genera with $\geq 4 \%$ abundance, Eubacterium was inversely correlated with oxalate levels and Escherichia showed a trend towards an inverse correlation with citrate levels.

"Although our pilot studies were performed in a relatively small study group the comparative analysis of the GMB from stone formers and non-stone formers would provide the first published evidence that kidney stone patients might have a distinct gut microbial profile as compared with controls," say the authors. "Manipulation of the GMB has been shown to alter disease states like obesity and diabetes mellitus.... If a GMB signature could be identified in kidney stone patients, GMB manipulation might represent a novel preventative treatment for kidney stone disease."

Rebecca Kelsey

ORIGINAL ARTICLE Stern, J. M.et al. Evidence fo a distinct gut microbiome in kidney stone formers compared to non-stone formers. Urolithiasis http://dx.doi.org/10.1007/s00240-016-0882-9 (2016) 